\title{
Overview spectra and axial distribution of spectral line intensities in a high-current vacuum arc with $\mathrm{CuCr}$ electrodes
}

\author{
M. Lisnyak, ${ }^{\text {a) }}$ A. V. Pipa, S. Gorchakov, ${ }^{\text {b) }}$ S. Iseni, ${ }^{\text {a) }}$ St. Franke, A. Khapour, R. Methling, \\ and K.-D. Weltmann ${ }^{\text {b) }}$ \\ Leibniz-Institut für Plasmaforschung und Technologie e.V., Felix-Hausdorff-Straße 2, 17489 Greifswald, \\ Germany
}

(Received 27 July 2015; accepted 15 September 2015; published online 29 September 2015)

\begin{abstract}
Spectroscopic investigations of free-burning vacuum arcs in diffuse mode with $\mathrm{CuCr}$ electrodes are presented. The experimental conditions of the investigated arc correspond to the typical system for vacuum circuit breakers. Spectra of six species $\mathrm{Cu}$ I, Cu II, Cu III, Cr I, Cr II, and Cr III have been analyzed in the wavelength range $350-810 \mathrm{~nm}$. The axial intensity distributions were found to be strongly dependent on the ionization stage of radiating species. Emission distributions of $\mathrm{Cr}$ II and $\mathrm{Cu}$ II can be distinguished as well as the distributions of $\mathrm{Cr}$ III and $\mathrm{Cu}$ III. Information on the axial distribution was used to identify the spectra and for identification of overlapping spectral lines. The overview spectra and some spectral windows recorded with high resolution are presented. Analysis of axial distributions of emitted light, which originates from different ionization states, is presented and discussed. http://dx.doi.org/10.1063/1.4931766
\end{abstract}

\section{INTRODUCTION}

Vacuum circuit breakers are widely used as switching devices in medium voltage electrical networks. Optimization of such devices for the needs of modern network systems, such as an extension of voltage range and operation at higher currents, requires deeper understanding of physical processes which determine the interruption processes.

During the arc operation, the cathode spots are the main source of the evaporated material in vacuum arcs. At currents above $1 \mathrm{kA}$, the whole surface of the cathode is covered with spots, which occur simultaneously and feed the plasma expanding into the gap. ${ }^{1,2}$ When the current exceeds a certain value, constriction of the arc in the vicinity of the anode appears, and the anode spot is formed. It produces additional intensive metallic vapor in addition to the cathode spots and might impede current interruption at current-zero. ${ }^{3}$ This operation mode should be avoided for successful interruption. If the anode spot does not appear, the anode is still a source of material but the vaporization rate is much lower. ${ }^{3,4}$

For deeper understanding of basic physical processes in vacuum arcs plasma parameters are needed. High current vacuum arcs (HCVA) are intensively studied theoretically and experimentally. ${ }^{5-9}$ As a method of choice, optical emission spectroscopy (OES) is a noninvasive technique to get better insight into the plasma. Development of vacuum circuit breakers raised interest to systematic spectroscopic investigation of the vacuum arc between $\mathrm{Cu}$ and $\mathrm{Cu}$-composed electrodes. Spectroscopic investigations of the vacuum arc with $\mathrm{Cu}$ electrodes are presented in works, ${ }^{10-15}$ where plasma parameters were determined. The number of publications dealing with $\mathrm{Cu}$ spectra description is rather limited. ${ }^{10,11,13,15-19}$ Most of them have been studied under low current conditions, 100-400 A

\footnotetext{
${ }^{a)}$ Present address: GREMI, UMR 7344, CNRS/Université d'Orléans, BP 6744, 45067 Orléans Cedex 2, France.

${ }^{b}$ Authors to whom correspondence should be addressed. Electronic addresses: gorchakov@inp-greifswald.de and weltmann@inp-greifswald.de
}

(Refs. 16-19) and 20-1000 A. ${ }^{11}$ Some of the spectral lines in the UV range are mentioned by Schellekens, ${ }^{15}$ while the plasma temperature and density were determined from the corresponding line intensities. Spectroscopic methods for HCVA investigations were applied in order to obtain the excitation temperature of $\mathrm{Cu}$ I, Cu II by several authors. ${ }^{10,13}$

For the application of the plasma characterization methods based on OES spectral line identification should be performed primarily. The $\mathrm{Cu}$ spectrum is densely populated with lines and contains many transition in abundance, ${ }^{20-25}$ which often is the reason for overlapping of the lines and makes the line identification challenging. ${ }^{26}$ The use of the alloys causes additional difficulties due to appearance of characteristic lines of each alloy component. Analysis of metallic spectra is often non-trivial, especially in case of nonstationary, non-equilibrium discharge such as vacuum arcs, which requires the measurements with high temporal, spatial, and spectroscopic resolution. The present work introduces the results of a spectroscopic investigation of the HCVA with $\mathrm{CuCr}$ electrodes. The aim of the work is to perform spectral line identification of the arc radiation in the wavelength range $350 \mathrm{~nm}-810 \mathrm{~nm}$. Spatially resolved spectra have been analyzed along the symmetry axis of a cylindrical electrode system.

\section{EXPERIMENTAL SETUP}

Experimental setup ${ }^{27}$ consists of high-current generator, model vacuum switch, and diagnostics equipment. The generator module consists of capacitors, inductors as well as charging and control units which are connected to a high speed and high resolution measuring system. There are 30 capacitors of $525 \mu \mathrm{F}$ each. They are divided to 5 parallel sections. There are 5 inductors of $4700 \mu \mathrm{H}$ which are combined in series with each capacitor group. To generate a $50 \mathrm{~Hz}$ AC waveform, this combination is in series with an inductor of $2730 \mu \mathrm{H}$. The capacitors can be charged up to maximum 


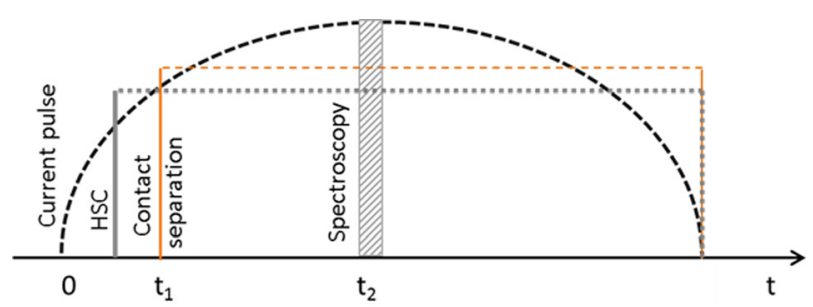

FIG. 1. Chronogram of the experimental setup and diagnostic apparatus. Time moment $\mathrm{t}=0 \mathrm{~s}$ corresponds to the beginning of the current pulse; HSC is time when the high-speed camera acquisition starts; $t_{1}$ is the time moment of the contact separation; $t_{2}$ is the beginning of the ICCD camera acquisition, with $200 \mu$ s duration.

$18 \mathrm{kV}$ to generate a $15 \mathrm{kA}$ in peak $\mathrm{AC}$ waveform with a frequency of $50 \mathrm{~Hz}$. The LC elements with constant capacity of $262.5 \mu \mathrm{F}$ and stepwise decreasing inductances are coupled in series to produce rectangular pulses. The capacitors can be charged up to $18 \mathrm{kV}$ to generate a $5 \mathrm{kA}$ DC waveform over $10 \mathrm{~ms}$. The model vacuum switch consists of a vacuum chamber, an electrode system, and pneumatic mechanical actuator.

The vacuum chamber is equipped with flat glass windows (BK7) for spectroscopic investigations and study of the arc dynamics. The used pumping system provides pressures below $10^{-7}$ mbar. CuCr cylindrical electrodes of $20 \mathrm{~mm}$ diameter have been used. One of the electrodes is movable similar to commercial circuit breakers. In the present experiment, the cathode is moveable with constant velocity of about $1 \mathrm{~m} / \mathrm{s}$. The arc is initiated by the separation of the electrical contact while a current pulse is applied.

The optical diagnostics system consisted of a high-speed camera Redlake MotionPro Y6 for visualization of arc dynamics. Spectroscopic measurements have been performed with a spectrograph Acton SP2500 (0.5 m focal length) and combination of deflecting and focusing mirrors with 1:1 magnification ratio. The spectrometer is equipped with two gratings. For the acquisition of the overview spectra, the $150 \mathrm{~g} / \mathrm{mm}$ grating allowing for a spectral window of about $120 \mathrm{~nm}$ width has been used. To achieve a higher spectral resolution (about $0.05 \mathrm{~nm}$ ), a grating with $1800 \mathrm{~g} / \mathrm{mm}$ and an entrance slit of $50 \mu \mathrm{m}$ were used. This parameter set
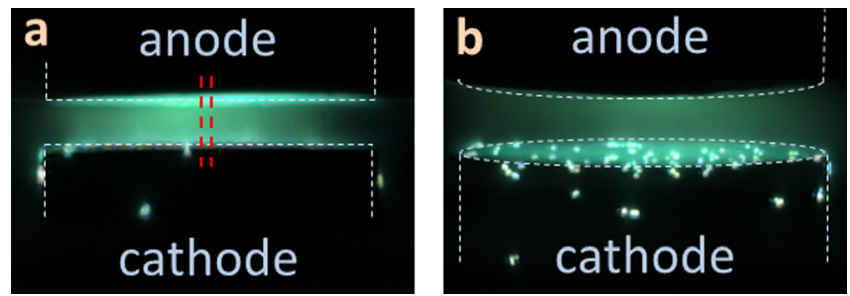

FIG. 2. Images of the arc from the high speed camera. $I=2.3 \mathrm{kA}, \mathrm{h}=4.5 \mathrm{~mm}$, exposure time $20 \mu \mathrm{s}$. Red dashed lines show the projection of the spectrograph slit.

corresponds to a spectral window of $12 \mathrm{~nm}$. Registration of acquired spectra was performed by an intensified chargecoupled device (iCCD) camera (Princeton Instruments PIMAX4, $1024 \times 1024$ pixels). The relative spectral sensitivity calibration of this configuration of the spectrograph is carried out using a tungsten strip lamp (OSRAM Wi 17/G). The high-speed camera was operated with frame rate of 20000 $\mathrm{fr} / \mathrm{s}$ and exposure time of $20 \mu \mathrm{s}$.

Figure 1 shows the synchronization timeline. The beginning of the electrode separation is further referred as moment $t_{1}$. In the case of sine-wave operation, the current reaches its maximum value at about $5 \mathrm{~ms}$. The electrode distance is approximately $\mathrm{h}=4.5 \mathrm{~mm}$. This instant $\left(\mathrm{t}_{2}\right)$ is chosen for the spectroscopic investigation. The photographs of the arc at the instant $t_{2}$ are presented in Figure 2.

The spectrograph slit is placed in such a way that the spectral information from the region between the electrodes and perpendicular to the electrode surface was gathered. Examples of the spectral resolved images are shown in Figure 3.

Both acquisition systems are synchronized with the separation of the electrodes to insure the reproducibility of the measurements. The spectrum acquisition starts at instant $t_{2}$ with a typical exposure time of $200 \mu \mathrm{s}$.

\section{RESULTS}

\section{A. Spectra of high-current vacuum arc (HCVA)}

The diffuse arc column occupies the whole inter-electrode gap as shown in Figure 2(a). The cathode spots are distributed
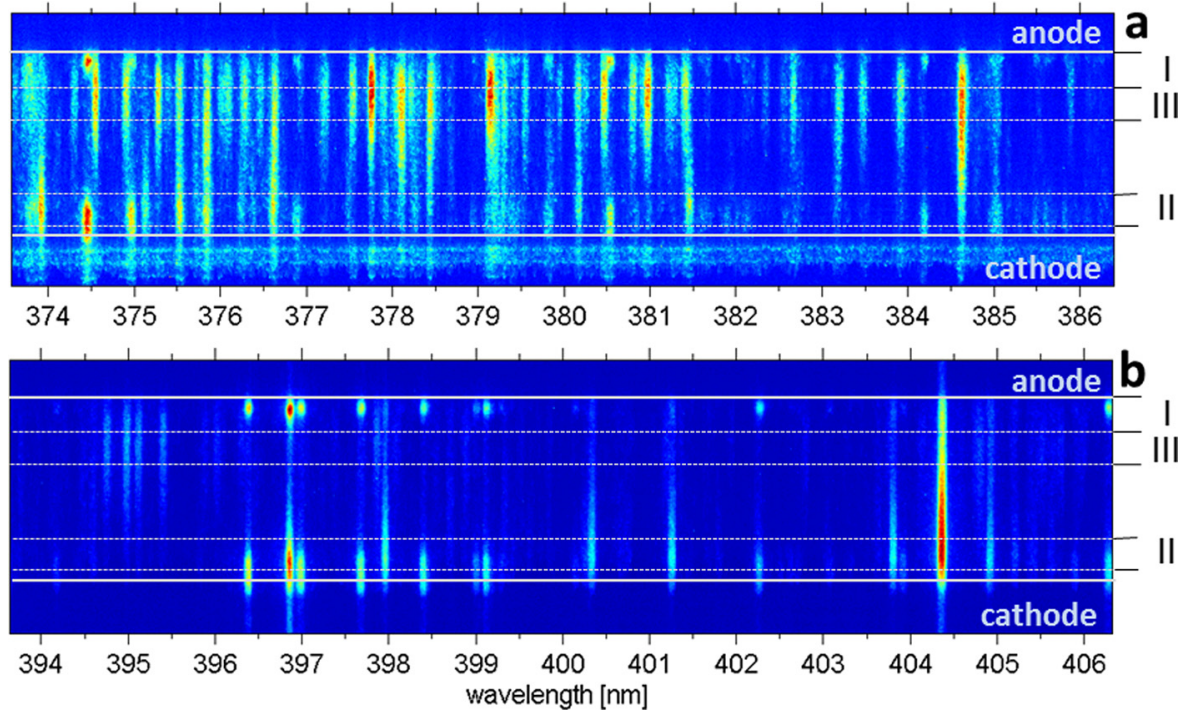

FIG. 3. Spectrum of HCVA, $I=2.3 \mathrm{kA}$ $\mathrm{h}=4.5 \mathrm{~mm}$, exposure time $200 \mu \mathrm{s}$ (a) central wavelength $380 \mathrm{~nm}$, (b) central wavelength $400 \mathrm{~nm}$. The solid horizontal lines mark positions of electrodes edges. Dashed lines show the boundaries of three specific different zones. 
stochastically on the cathode surface (Figure 2(b)). Due to the absence of an external magnetic field, some of the spots are moving to the lateral side of the electrode. During the spectral acquisition, the positions of the electrodes are determined on the OES iCCD images (Figure 3). The horizontal solid lines indicate the electrode edge position in Figure 3. The cathode position is determined with accuracy of about $0.25 \mathrm{~mm}$. The position of the cathode spots may appear aside of the line which defines the mean cathode surface location. A cathode spot in the spectrometer field of view can be recognized by a strong continuum radiation below the cathode edge position as in the upper image of Figure 3. The emission outside of the gap has not been considered for analysis.

The arc column visually appears nearly homogenous, however, spectrally resolved images indicate that the intensity distribution along the axis strongly depends on the wavelength (Figure 3). In order to simplify the interpretation of the complex spectroscopic structure of the spectrum, the inter-electrode distance was divided in three zones of approximately equal length: (I) anode zone, (II) cathode zone, and (III) arc column as shown in Figure 3. For each zone, the signal is spatially averaged in order to improve the signal-to-noise ratio.

Figure 4 presents an overview spectrum made of four spectral windows measured with low spectral resolution. The discontinuities between the measured spectral windows (shotto-shot fluctuations) are compensated by normalization on the overlapping part of the spectra. The spectra of each zone are presented with different line style. The figure is scaled to demonstrate the complexity of the spectral distribution and thus the intensities of the strong lines from zone I are cut out. The qualitative difference between the spectra emphasizes that consideration of the axial distribution is important for the identification of the abundance of the spectral lines. The complex spectral structure arises from the overlap of many spectroscopic lines and makes the line identification a difficult task. Furthermore, the spectrum changes for various axial positions and different lines can dominate in the same spectral interval. For the identification of the spectra of HCVA with $\mathrm{CuCr}$ electrodes sufficient spectral and spatial resolution is needed.

The high resolution spectra $(50 \mathrm{pm})$ are presented in Figure 5. Each spectroscopic window presents the spectra of three inter-electrode zones with the same line style as in Figure 4. Well separated single spectral lines are marked with element label, its ionization state and corresponding wavelength in nm. Different elements $\mathrm{Cu}$ and $\mathrm{Cr}$ are distinguished with italic and bold text format. In the case of overlap of two spectral lines belonging to the same element, for the second spectral line the distinguishing digits of the wavelength after decimal point are shown. For example $\mathrm{Cr} \mathrm{I}$ $529.73-.89$ indicates Cr I 529.73 and Cr I 529.89 spectral lines. In more complex cases, the element symbol and ionization stage is shown without wavelength. Spectral lines which are not presented in known tables ${ }^{20-25}$ but observed during the experiment are labeled with question mark "?." For most of them according to the axial profile, it is possible to speculate which element and ionization state they are, therefore after "?" placed ionization state with corresponding text style (italic for $\mathrm{Cu}$; bold for $\mathrm{Cr}$ ). For example "? III," the most presented amount undefined lines, is supposed to be double charged ionic line of $\mathrm{Cu}$.

\section{B. Axial distributions}

In the previous work, ${ }^{27}$ it was noted that plasma species (atoms and ions) show well distinguished axial distributions of the intensities. The axial distributions of different spectral lines integrated over the spectral line profile are normalized to the maximum value of their spatial distributions, the results are shown in Figure 6-9. For each distribution, the wavelength (in $\mathrm{nm}$ ) of the spectral line and corresponding energy level of upper energetic state (in $\mathrm{eV}$ ) are assigned. The distributions are derived from high resolution spectra (like, e.g., Fig. 5), which are obtained for the three different zones. Position $\mathrm{z}=0 \mathrm{~mm}$ corresponds to the anode edge. The position of the cathode is indicated by the shaded rectangle, because the cathode is moving. The width of the rectangle reflects the cathode travelling distance during the spectral acquisition.

Figure 6 presents thee axial distributions of the atomic line intensities ( $\mathrm{Cr} \mathrm{I}$ and $\mathrm{Cu} \mathrm{I}$ ). They are characterized by intensive radiation peaks near the electrodes and lower intensity in the gap. Due to spatial resolution of the optical system of about $0.5 \mathrm{~mm}$, the radiation profiles in vicinity of the electrodes are broader than expected. Near the cathode, an additional profile smoothing is caused by uncertainty in cathode position due to the movement during the contact separation and statistical behavior of cathode spots on the cathode surface and on the cathode lateral surface.

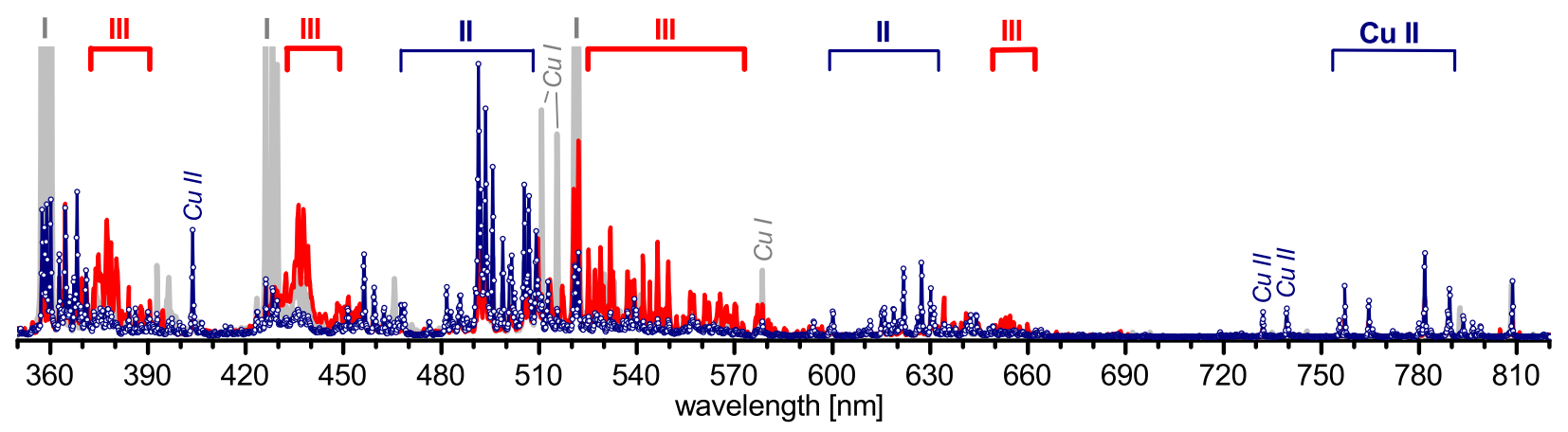

FIG. 4. Overview spectrum of HCVA with CuCr electrodes, I $=2.3 \mathrm{kA}, \mathrm{h}=4.5 \mathrm{~mm}$, gate $200 \mu$ s. The light grey line-represents spectrum of zone I (near anode), the blue line with open circles corresponds to zone II (near cathode) and red ones to zone III. The assignments of the inter-electrode zones are shown in Figure 3. 

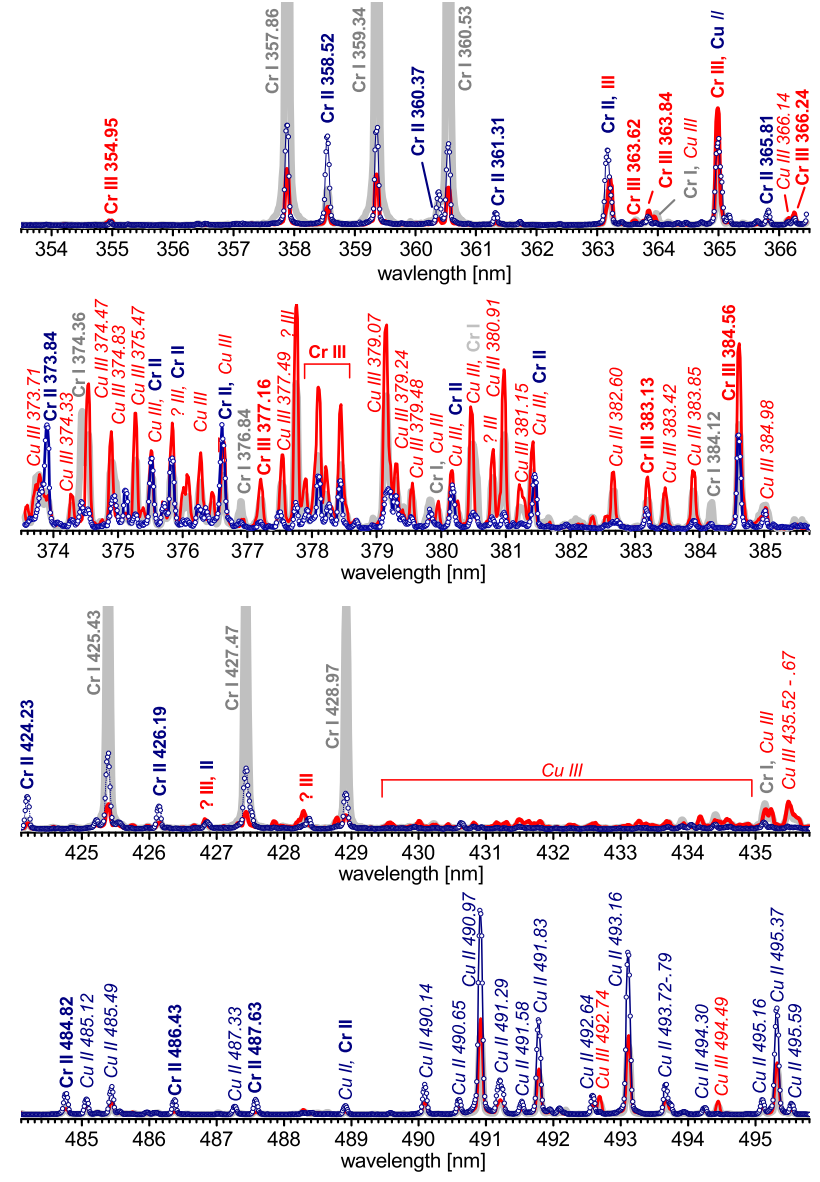

(a)
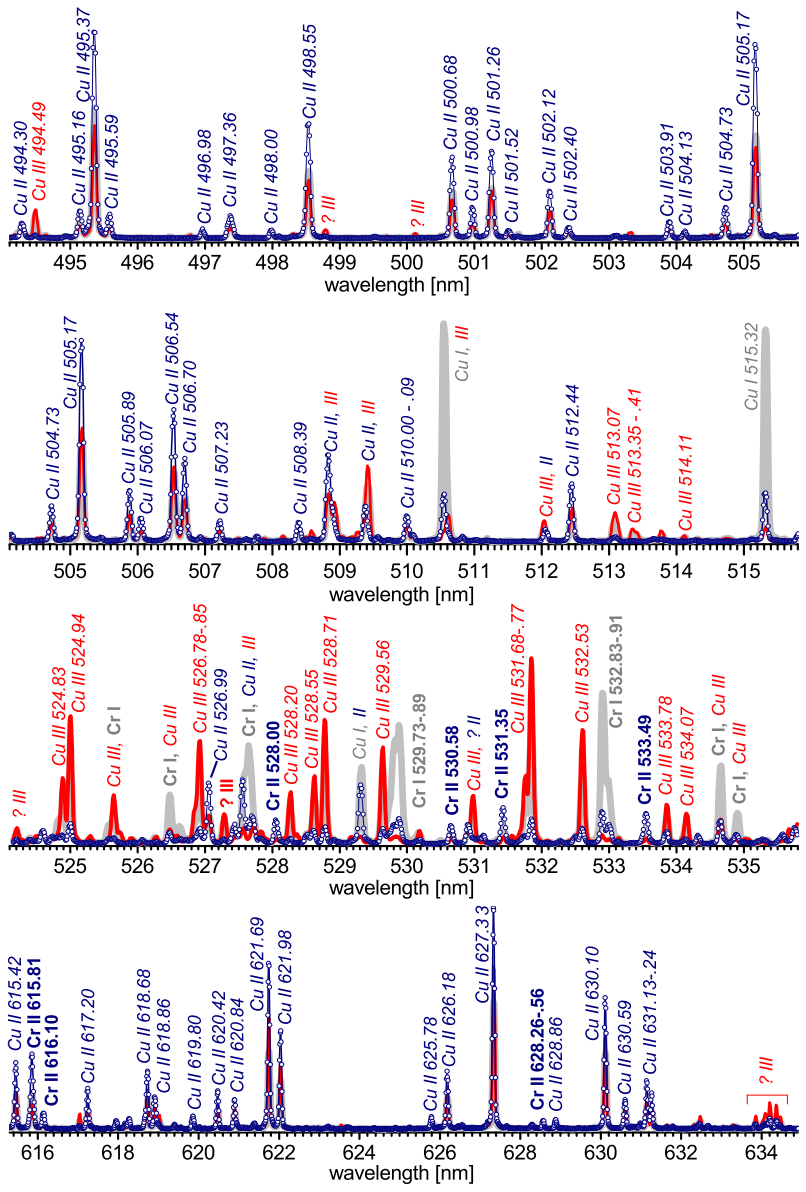

(b)

FIG. 5. High resolution spectra of HCVA with $\mathrm{CuCr}$ electrode, $\mathrm{I}=2.3 \mathrm{kA}, \mathrm{h}=4.5 \mathrm{~mm}$, gate $200 \mu \mathrm{s}$. Same line styles as in Figure 4.

In Figures 7 and 8, the axial distributions of intensities for single and double charged ions are shown. Axial distributions have been acquired for most intense spectral line in the chosen spectral windows (see Fig. 5). The deviation of the intensity distributions is related to the reproducibility of the experimental conditions, like, e.g., instant of the arc ignition. Differences between distributions near the cathode area emphasize statistical random appearance of the cathode spot processes and require more detailed investigations.

Single charged ions show a great number of well resolved spectral lines with strong intensities. Axial distributions of $\mathrm{Cr}$ II look smoother and show better reproducibility
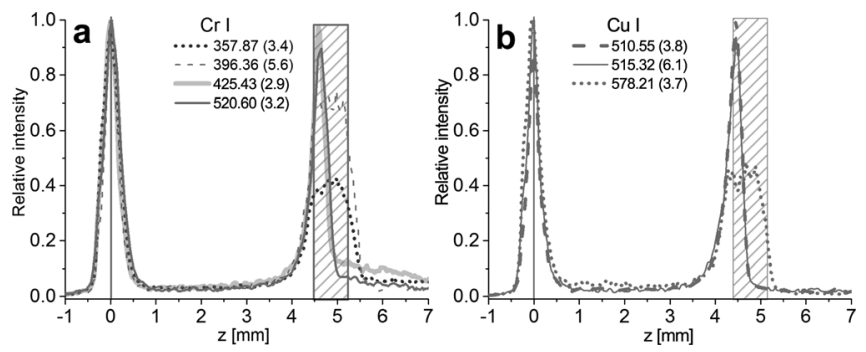

FIG. 6. Relative intensity distribution of the atomic spectral lines along the arc axis. (a) $\mathrm{Cr} \mathrm{I}$, (b) CuI. $\mathrm{z}=0 \mathrm{~mm}$ is anode position, shaded area at $\mathrm{Z}=4.5-5.2 \mathrm{~mm}$ is the position of the cathode during exposure time. for different shots in comparison to distributions of $\mathrm{Cu}$ II (Fig. 7). The intensity fluctuation can be therefore used as an additional criterion for the line identification.

Axial profiles of Cr III are very noisy (Fig. 8). There are only few Cr III lines which can be selected for investigations, however, they have a low intensity and are emitted in the UV range. Intensity of $\mathrm{Cu}$ III lines is also weak; however, there is a larger choice of well separated lines in the visible range. The experiments show that an increase of the current leads to immediate intensity growth of double charged ion lines.

The information about the axial distributions of the intensities of different species for arc current $\mathrm{I}=2.3 \mathrm{kA}$ is summarized in Figure 9. Distributions for ions can be well distinguished. The first maximum for $\mathrm{Cu}$ II is shifted to the
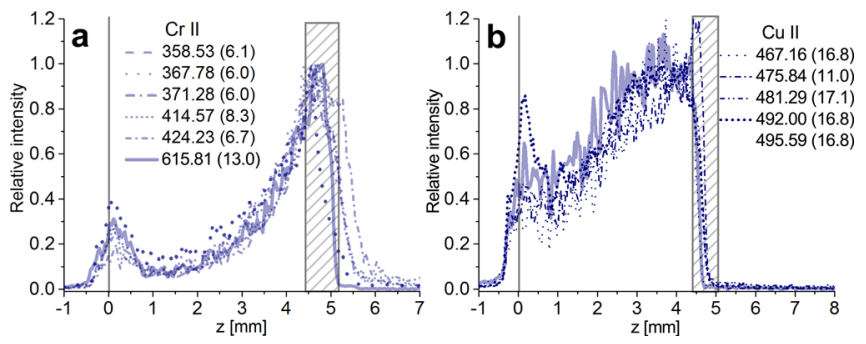

FIG. 7. Same as Fig. 6 but for the ions $\mathrm{Cr}$ II (a) and $\mathrm{Cu}$ II(b).

This is the Accepted Manuscript version -post-print- of an article accepted for publication in Journal of Applied Physics.

This article may be downloaded for personal use only. Any other use requires prior permission of the authors and AIP Publishing. This article may be found at doi:10.1063/1.4931766.

AIP Publishing is not responsible for any errors or omissions in this version of the manuscript or any version derived from it. 


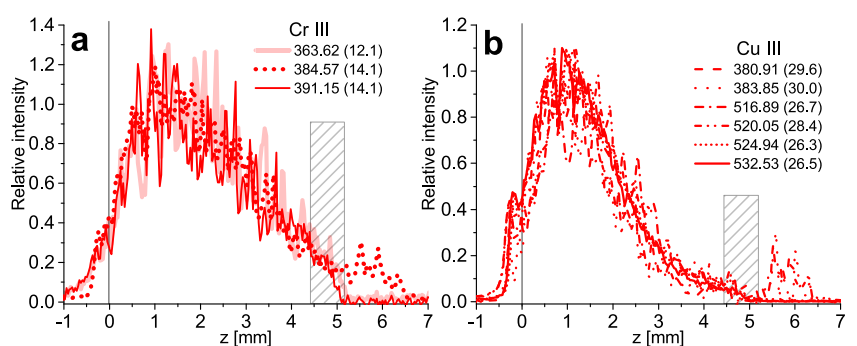

FIG. 8. Same as Fig. 6 but for the ions Cr III (a) and Cu III(b).

arc column in comparison to Cr II. The distribution of the $\mathrm{Cu}$ III is narrower in comparison to Cr III. Close to the anode, the intensities are very sensitive to the axial position. Restricted spatial resolution of experimental setup makes the identification of the second maximum for single charged ion radiation near to anode complicated. Further investigations are necessary here. Three axial zones can be identified in accordance with maximum of radiation intensity for different ionization stages. The axial zones are marked I, II, and III in accordance to the ionization stage of the species. The comparison of the relative intensities acquired in indicated zones displays the dominant ionization stage in a certain spectral region. It is useful as for accurate identification on the basis of the high resolution spectra, Figure 5 as well as to infer low resolution overview spectrum (Fig. 4).

\section{Identification of the spectral lines}

Several data sources ${ }^{20-25}$ are used for spectral lines identification. Zaidel table of spectral lines ${ }^{25}$ is used for the intensive lines identification. Other spectral databases applied to complete the identification, such as Striganov, ${ }^{24}$ NIST, ${ }^{20}$ and Kurucz. ${ }^{21}$ These databases contain similar information for the majority of transitions. However, Refs. 21 and 24 include most transitions. Concerning the specific case of $\mathrm{Cu}$ II, the database ${ }^{24}$ is mostly used. Some data for the second ionization state for $\mathrm{Cu}(\mathrm{Cu}$ III) like the wavelength

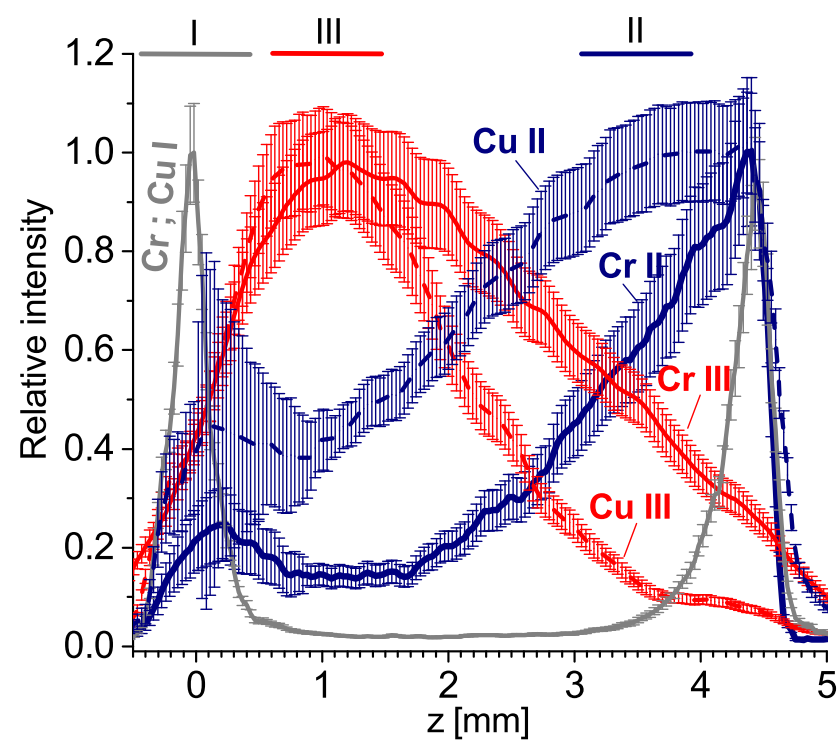

FIG. 9. Normalized intensity distribution of the spectral lines with respect to different ionization states of $\mathrm{Cu}$ or $\mathrm{Cr}$ along the arc axis. information energy levels of the transition for $\mathrm{Cu}$ III are presented in Ref. 23 only. The information about higher ionization states of $\mathrm{Cu}$, for instance, $\mathrm{Cu}$ IV can be found in Ref. 22. Comparison between these data and the results of current measurements confirms that such ionization state is not present in the plasma for studied parameter range.

Assignment of the spectral lines on the base of spectroscopic tables ${ }^{20-25}$ has been verified by axial distribution of the species emission. Unambiguous identification is required for obtaining axial intensity distributions of a single species. However, this is a quite difficult task since many lines are overlapping. Therefore, an iterative analysis of the experimental spectra has been applied. The final results of the axial distribution are shown in Figures 6-9. In the case of the overview spectrum, the isolated spectroscopic lines cannot be resolved (Fig. 4). However, it is possible to distinguish the spectral regions where the line intensities which belong to certain ionization state are dominating. These regions are marked on the top of Figure 4 with I, II, and III corresponding to the ionization state. For instance, $\mathrm{Cu}$ II lines are well isolated in the region of $720-810 \mathrm{~nm}$, even when it is necessary to pay attention to the emission of atomic lines of $\mathrm{Cr}$ I at $746.23 \mathrm{~nm}$ and $\mathrm{Cu} \mathrm{I}$ at $793.31 \mathrm{~nm}$. Similar situation is observed with intensity of $\mathrm{Cu}$ II lines in the range 490-509 nm, However, it should be noted that in this region, few week lines of $\mathrm{Cu}$ III (494.49 nm and $500.13 \mathrm{~nm}$ ) occur which could become more intense for higher currents.

\section{Parameters variation}

The axial distributions have been studied for different parameters, namely, current, distance between electrodes, and contact separation velocity. Due to the interdependent nature of the latter parameters to influence each other and thus contribute to the global behavior of the investigated system. ${ }^{3}$ Therefore, it is convenient to fix one of the parameter and to make a variation of the others. A DC current pulse with the possibility to change current amplitude has been used.

A spectral interval $505-520 \mathrm{~nm}$ was chosen for study of parameter variation. It contains well resolved spectral lines of different ionization states of $\mathrm{Cu}$, among which were chosen $\mathrm{Cu}$ I $510.55 \mathrm{~nm}, \mathrm{Cu}$ II $508.84 \mathrm{~nm}, \mathrm{Cu}$ III $513.07 \mathrm{~nm}$ for the study of the axial distributions. Spectral acquisition in different time instants corresponds to spectra of the arc with different length. Figure 10 shows the axial distribution acquired with fixed value of DC current $\mathrm{I}=2.3 \mathrm{kA}$ for the arc length $\mathrm{h}=1.2 \mathrm{~mm}, 2.2 \mathrm{~mm}, 4 \mathrm{~mm}$, and $4.8 \mathrm{~mm}$.

The axial distributions have similar shapes and qualitatively coincide with distributions of $\mathrm{Cu}$ lines from Figure 9 scaled according to the distance between the electrodes. Maximum intensities have been observed in the close vicinity to the electrodes.

Variations of the contact separation speed in the range of $1 \mathrm{~m} / \mathrm{s}-2 \mathrm{~m} / \mathrm{s}$ with $\mathrm{I}=2.3 \mathrm{kA}$ have been investigated as one of the parameter. The analysis clarifies that the axial distributions have the same behavior as in Figure 10 for the same instantaneous electrode distance.

For a vacuum arc variation of the current in significant range is not possible due to the appearance of instabilities. 

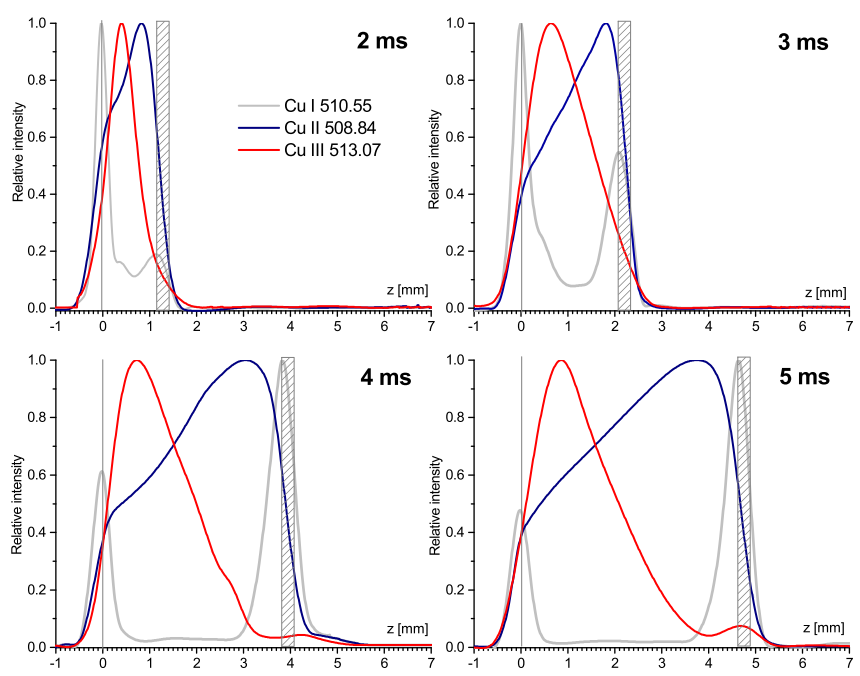

FIG. 10. Normalized intensity distributions of the spectral lines with respect to different ionization states of $\mathrm{Cu}$ obtained at different instants at fixed current of $2.3 \mathrm{kA}$ at different instants $\mathrm{t}_{2} 2 \mathrm{~ms}, 3 \mathrm{~ms}, 4 \mathrm{~ms}$, and $5 \mathrm{~ms}$.

Therefore, values of current have been investigated in range of $1 \mathrm{kA}-2.3 \mathrm{kA}$ when the anode spot is not presented yet. The lower currents are not presented due to low signal to noise ratio for spectroscopic investigations, so far for the currents less than $2 \mathrm{kA} \mathrm{Cu}$ III spectral lines are weak and not appropriative for investigations. Axial distributions of $\mathrm{Cu} \mathrm{I}$ and $\mathrm{Cu}$ II lines for different currents are presented in Figure 11, where different line styles correspond to different currents and different color to different ionization state.

For a fixed current value, the axial distributions do not change qualitatively. The spatial distribution stretches and shrinks along the electrode gap. For the current values above $2 \mathrm{kA}$, the axial distribution has similar proportion, i.e., high intensities of $\mathrm{Cu} \mathrm{I}$ in the vicinity of the electrodes and low intensities in the gap. It is interesting to note that intensity maximum of $\mathrm{Cu}$ I decreases with increase inter-electrode gap (Figure 11). The axial distributions of $\mathrm{Cu}$ II have the maximum near the cathode and smoothly decrease in the gap. Opposite situation for $\mathrm{Cu}$ II takes place for arcs with
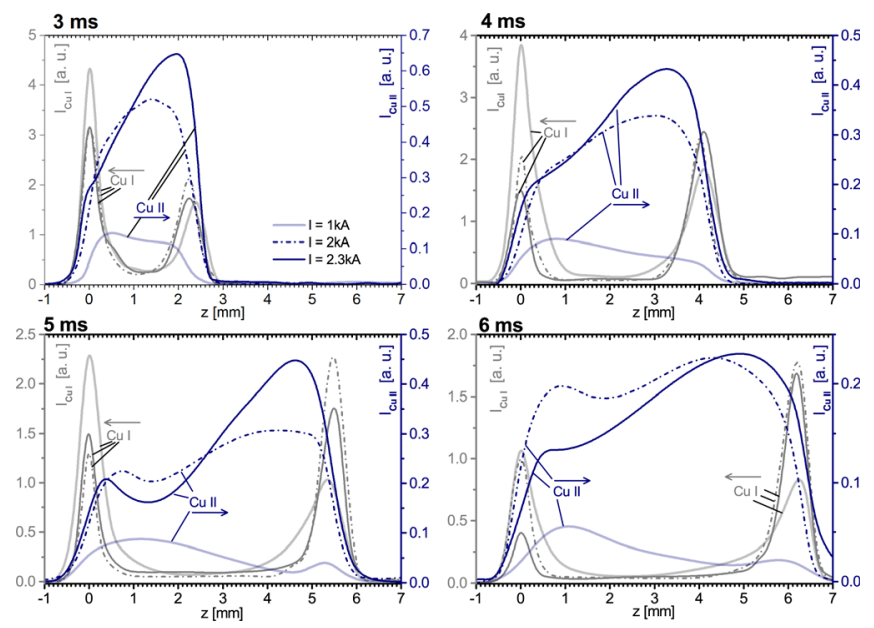

FIG. 11. Intensity distributions of the spectral lines with respect to different ionization states of $\mathrm{Cu}$ obtained for different currents in DC mode $\mathrm{I}=1 \mathrm{kA}$, $2 \mathrm{kA}$, and $2.3 \mathrm{kA}$ and at different instants $\mathrm{t}_{2} 3 \mathrm{~ms}, 4 \mathrm{~ms}, 5 \mathrm{~ms}$, and $6 \mathrm{~ms}$.
$\mathrm{I}=1 \mathrm{kA}$, when the maximum of $\mathrm{Cu}$ II intensity occurs near the anode side. Meanwhile, $\mathrm{Cu}$ I distributions decrease smoothly at the maximum at the electrode edges into the gap. The maximum of $\mathrm{Cu}$ I radiation occurs at the anode side.

Results of investigations in DC conditions show that for different delay time, i.e., different electrode gaps, the distributions are similar but scaled according to the gap length (Fig. 10). The width of the near electrode zones depends on experimental parameters, like, e.g., current and electrode material, and remains approximately unchanged for different distances between electrodes. The bulk length increases with growing electrode gaps. Such behavior can draw an analogy between vacuum arc and glow discharge with its well-known structure.

Variation of the current density shows that axial distributions are changing (Fig. 11). However, they keep the scaling behavior for different electrode distances. Most significant changes concern $\mathrm{Cu}$ II intensity distribution, the maximum of distribution for current $\mathrm{I}=1 \mathrm{kA}$ is placed near anode, opposite to the case with $\mathrm{I}=2.3 \mathrm{kA}$. The intensity distributions of $\mathrm{Cu} \mathrm{I}$ have a larger decay length from the cathode side into the gap. Intensity of double ions is very weak and could not be detected due to low signal to noise ratio. Thus for low current densities (in our case it is $0.35 \mathrm{kA} / \mathrm{cm}^{2}$ ) ionization degree of the plasma into the gap is lower, the reason could be lower electron temperature in the plasma bulk for low current densities. Increasing current leads to appearance of spectral lines of double charged ions with maximum of the intensity near anode, while $\mathrm{Cu}$ II has maximum of intensity near the cathode and decreasing of intensity with distance from the cathode. Such behavior can be the result of ionization processes in plasma column from single ions to double ions due to high electron temperature.

\section{DISCUSSIONS}

A limited number of studies dealing with spectral line identification in vacuum arcs with $\mathrm{Cu}$ electrodes have been found in the literature. ${ }^{11,16-19}$ The first work which contains tables of optical transitions of copper spark in vacuum arc was published by Ravary and Charles, ${ }^{16}$ where lines of $\mathrm{Cu} \mathrm{I}$, $\mathrm{Cu}$ II, and $\mathrm{Cu}$ III were identified in $\mathrm{UV}$ and visible range $(240-660 \mathrm{~nm})$. Spectra for pure $\mathrm{Cu}$ electrodes have been studied under low current conditions, $100-300 \mathrm{~A},{ }^{16-18}$ 175-340 A, ${ }^{19}$ and 20-1000 A. ${ }^{11}$ Some of them report spectra in a wide wavelength range ${ }^{17,18}$ with quite low spectral resolution (around $1 \mathrm{~nm}$ ) from which it is possible to recognize the $\mathrm{Cu} \mathrm{I}$ and $\mathrm{Cu}$ II lines only. Practical use of the tables and low resolution spectra is problematic, especially when the spatial and time resolution is disregarded. Thus it is difficult to correlate the results of present work with previously measured spectra. However few comments can be made.

Abundance of $\mathrm{Cu}$ I spectral lines ${ }^{11,18}$ result from quite long acquisition times during and after the current pulse. In pulsed current regime, the atomic line radiation has much longer decay time in comparison with ion emission. ${ }^{18}$ Slower decay of the atomic emission was also detected in case of Ga electrodes. ${ }^{28}$ In the present work, the spectrum 
was recorded at the peak value of the current and only strongest $\mathrm{Cu}$ I lines can be recognized. Significant emission of the $\mathrm{Cu}$ III (unlike to the previous works ${ }^{11,16-19}$ ) could be a result of higher arc current. Increasing of $\mathrm{Cu}$ III intensities with arc current have been demonstrated in Ref. 14 . Significant contribution of $\mathrm{Cu}$ III emission results in very complex spectra. Axial distributions presented for different species show local maxima at specific spatial positions (Fig. 9). Intensity maxima for atomic lines occur close to the electrodes. At the small distance $(0.7-1 \mathrm{~mm})$ from the cathode, a first maximum of singly ionized ions appears. The maximum of the double charged ions appears at further away from the cathode. Similar axial behavior for atomic and ionic states was found in vacuum arcs with $\mathrm{Al}, \mathrm{Zn}$, and Ga-In electrodes. $5,29,30$

Images of HCVA with $\mathrm{CuCr}$ electrodes in spectral intervals selected by interference filters $425 \pm 5 \mathrm{~nm}, 497 \pm 5 \mathrm{~nm}$, and $625 \pm 5 \mathrm{~nm}$ have been presented in Ref. 31 . The bright plume near the anode observed in the wavelengths ranges $425 \pm 5 \mathrm{~nm}$ and $497 \pm 5 \mathrm{~nm}$ we attribute to the presents of $\mathrm{Cu}$ III spectral lines. The current investigations show that in both spectral regions spectral lines of $\mathrm{Cu}$ III are present, see Figure 5, moreover for chosen current conditions as in Ref. $31(\mathrm{I}=15 \mathrm{kA})$ intensity of $\mathrm{Cu}$ III should be dominant, since intensity of $\mathrm{Cu}$ III spectral lines increases with increasing current density. Thus, the axial intensity distribution presented in this work does not contradict with the observation reported in Ref. 31.

Finally it can be concluded that axial intensity distributions are specific for each type of the emitting species. The phenomena can be observed in a wide range of the experimental condition rather than being unique for the used experimental setup. It should reflect basic physical properties of the vacuum arc. It is well known that the cathode spots are the source of both ions and atoms. ${ }^{32}$ Consequently, an intense radiation of ions and atoms should occur near the cathode. The rapid decline of atomic radiation in this region is a result of fast ionization processes, which lead to rapid increase of $\mathrm{Cu}$ and $\mathrm{Cr}$ ions in vicinity of the cathode (Fig. 9). ${ }^{33}$

The ions originating from the cathode spots have axial velocity about $10^{6} \mathrm{~cm} / \mathrm{s}$ (Ref. 34 ) and are able to cover the large distances within the gap. Nevertheless, the intensity of the single charged ion spectral lines is decreasing with distance from the cathode, while the intensity of double charge ions is increasing. The authors of Refs. 5 and 29 explain this fact with a high electron temperature (about $6 \mathrm{eV}$ ), which was obtained by calculations ${ }^{6}$ and measured by electrical probes in Ref. 35, where also shown that electron temperature grows with the distance from the cathode to anode.

The increase of atomic radiation near the anode surface is caused by the emission of atoms by the anode. ${ }^{6,12,19,36}$ The mechanisms of such emission have different sources, for example, the evaporation due to heating of the anode surface by arc itself ${ }^{6}$ or material sputtering. ${ }^{36}$ The atoms emitted by the anode become ionized in the vicinity of the anode and form "slow" ions. ${ }^{12}$ Existence of a second maximum near the anode in axial distribution (Fig. 9) for ionic component can be the result of those processes.
TABLE I. Energy of the upper emitting level $(E)$, ionization energy $\left(E_{\mathrm{i}}\right)$ and position of the intensity distribution maximum as a distance from anode (d) taking from Figure 7. *-corresponds to spectral lines which exist only in Striganov ${ }^{24}$ database.

\begin{tabular}{lccc}
\hline \hline Species & $\mathrm{d}(\mathrm{mm})$ & $\mathrm{E}_{\mathrm{i}}(\mathrm{eV})$ & $\mathrm{E}(\mathrm{eV})$ \\
\hline $\mathrm{Cr}$ I & $0 ; 4.5$ & 6.77 & {$[2.9-7.8]$} \\
$\mathrm{Cu}$ I & $0 ; 4.5$ & 7.73 & {$[3.8-7.7]$} \\
$\mathrm{Cr}$ II & 4.3 & 16.50 & {$[6-7] ;[8-10] ;[13-14]$} \\
$\mathrm{Cu}$ II & 3.9 & 20.29 & {$[10-11]^{*} ;[15-18]$} \\
$\mathrm{Cr}$ III & 1.4 & 30.96 & {$[11-14]$} \\
$\mathrm{Cu}$ III & 1.1 & 36.83 & $26-30$ \\
\hline \hline
\end{tabular}

Table I summarizes the data of the local maximum position, ionization energy and range of energy levels from which the intense line radiation occurs for spectral lines in range $350-810 \mathrm{~nm}$. The energies corresponding to the wavelengths of line emission belong to the range from $1.8 \mathrm{eV}$ to $3.4 \mathrm{eV}$. However, the upper energetic levels are spread over a large range of energies, starting from $2.9 \mathrm{eV}$ for the atomic line of $\mathrm{Cr}$ up to $30 \mathrm{eV}$ for the double charged ions of $\mathrm{Cu}$. Concerning the atomic emission of $\mathrm{Cr}$ and $\mathrm{Cu}$, their transitions results from the excitation of energetic levels distributed from $2.9 \mathrm{eV}$ to $7.8 \mathrm{eV}$ and constitute a relatively large range. Some of the $\mathrm{Cr}$ I lines even originate from autoionization levels. It might be remarked that $\mathrm{Cu}$ I emission is only observed from four district lines. Cr transitions originate from a wider scale of energy than $\mathrm{Cu}$. Furthermore, one can note that $\mathrm{Cu}$ II and $\mathrm{Cu}$ III transitions arise from energetic levels close to the ionization threshold, in contrast to Cr III, where the upper levels are well below the ionization energy. Indeed, emission lines of the latter ion are coming from energetic states $(11 \mathrm{eV}-14 \mathrm{eV})$ which have about half energy compared to $\mathrm{Cu}$ III transitions $(26 \mathrm{eV}-30 \mathrm{eV})$. Note that most of the transitions of $\mathrm{Cu}$ II are identified to come from energy levels ranging from $15 \mathrm{eV}$ to $18 \mathrm{eV}$, only few transition originates from the range $10 \mathrm{eV}$ to $11 \mathrm{eV}$, indeed they correspond to the spectral lines, identified with Striganov database. ${ }^{24}$

Remarkably, one can observe that the emission lines of different radiative species located at the same axial position originate from energetic levels with a large difference. For instance, in the middle of the gap, where radiation of $\mathrm{Cu}$ III and $\mathrm{Cr}$ III are dominating, energetic levels around $11 \mathrm{eV}$ to $14 \mathrm{eV}$ lead to $\mathrm{Cr}$ III emissions while the radiation of $\mathrm{Cu}$ III originates from energy states elevated from $26 \mathrm{eV}$ to $30 \mathrm{eV}$. Consequently, the coexistence of such difference in energy between excited species raises questions about the energy distribution within the gap. Although no explanation can be claimed, one can suppose in any event that the energy distribution may be entangled with the particle density distribution within the gap and/or with the spatial charge particle distribution.

\section{SUMMARY AND CONCLUSION}

The spectrum of the vacuum arc in chosen conditions contains atomic, ionic, and double ionic spectral lines of $\mathrm{Cu}$ and $\mathrm{Cr}$. There are several spectral lines which could not be 
identified according to the tables. ${ }^{20-25}$ Nevertheless, considering the axial distributions of these lines, they could be assigned to $\mathrm{Cu}$ III. The other elements as well as higher ionization states of $\mathrm{Cu}$ or $\mathrm{Cr}$ were not detected in the spectrum. Moreover, the continuum radiation is very weak and requires higher currents to reach intensities which can be evaluated for spectroscopic diagnostic of plasma parameters, e.g., electron density determination. ${ }^{14}$

In spite of visual homogeneity of the light along the discharge axis, a significant variation of the spectral lines intensity distribution in the gap has been observed. It was shown that each ionization state has its own specific spectral line intensity distribution in the gap. Each distribution exhibits a maximum. The maxima of atoms, ions, and double ion distributions are well distinguished and appear to have different position within the electrode gap. The structure of the axial intensities distributions is scaled with the inter-electrode distance; however, it depends qualitatively on discharge current.

Analysis of spectra along the axis gives some essential advantages in comparison with traditional approaches for arc analysis along the arc radius. The electrode gap can be divided into zones, where a spectrum is dominated by a selected ionization state. Such method allows for separating the line groups and may give an advice for identification of weak lines, unknown lines, and overlapping lines due to the huge amount of radiative transitions.

Low resolution overview spectrum indicates that it is possible to select wide wavelength ranges where emission of species in ionization stage of interest is dominating. This can enhance diagnostics of the discharge by means of fast camera imaging if the proper spectroscopic filters are used. The spectral windows with higher resolution are useful for preselection of the spectral range for diagnostics.

The separation of the emission belonging to species in different ionization stages along the inter-electrode distance can be seen from results of other works ${ }^{5,29,30}$ and can be related to basic properties of the HCVA without magnetic field. However, even qualitative interpretation of the phenomena is unsatisfactory at present time. Well distinguished difference in axial distribution of $\mathrm{Cu}$ II and $\mathrm{Cr}$ II ions as well as for $\mathrm{Cu}$ III and $\mathrm{Cr}$ III ions have been presented in addition and it calls for a qualitative theoretical description.

Detail analysis of the transition energies for the presented species in the spectra and comparing it with the corresponding axial distributions could arise the hypothesis about charged particle distribution in the gap. Based on the present work, further detailed investigations are necessary.
${ }^{1}$ R. L. Boxman, D. M. Sanders, and P. J. Martin, Handbook of Vacuum arc Science \& Technology: Fundamentals and Applications (William Andrew, 1996).

${ }^{2}$ J. M. Lafferty and J. D. Cobine, Vacuum Arcs: Theory and Application (Wiley, 1980).

${ }^{3}$ H. C. Miller, Contrib. Plasma Phys. 29(3), 223 (1989).

${ }^{4}$ S. M. Shkol'nik, Plasma Sources Sci. Technol. 20(1), 013001 (2011).

${ }^{5}$ R. L. Boxman and S. Goldsmith, J. Appl. Phys. 51(7), 3644 (1980).

${ }^{6}$ S. Goldsmith and R. L. Boxman, J. Appl. Phys. 51(7), 3649 (1980).

${ }^{7}$ A. Anders, IEEE Trans. Plasma Sci. 33(1), 205 (2005).

${ }^{8}$ L. Wang et al., J. Appl. Phys. 117(24), 243301 (2015).

${ }^{9}$ I. Zhirkov, E. Oks, and J. Rosen, J. Appl. Phys. 117(9), 093301 (2015).

${ }^{10}$ H. Toya, Y. Uchida, T. Hayashi, and Y. Murai, IEEE Trans. Plasma Sci. 14(4), 471 (1986).

${ }^{11}$ A. Anders and S. Anders, J. Phys. D: Appl. Phys. 24(11), 1986 (1991).

${ }^{12}$ A. L. V. Afanas'ev, N. Mitrofanov, and S. Shkol'nik, in Proceedings of the XIVth International Symposium on Discharges and Electrical Insulation in Vacuum, ISDEIV (Santa Fe, New Mexico, USA, 1990).

${ }^{13} \mathrm{~V}$. A. Bugaev et al., in Proceedings of the XXIIIth International Symposium on Discharges and Electrical Insulation in Vacuum, ISDEIV (Bucharest, Romania, 2008).

${ }^{14}$ A. A. Logatchev and M. V. Lisnyak, in Proceedings of the XXVth International Symposium on Discharges and Electrical Insulation in Vacuum, ISDEIV (Tomsk, Russia, 2012).

${ }^{15}$ H. Schellekens, Modelling of the Diffuse Arc in a Vacuum Circuit-Breaker (Technische Hogeschool Eindhoven, 1983).

${ }^{16}$ P. Ravary and B. Charles, J. Phys. D: Appl. Phys. 23(1), 123 (1990).

${ }^{17}$ D. Grondona, H. Kelly, A. Marquez, and A. Lepone, IEEE Trans. Plasma Sci. 30(1), 391 (2002)

${ }^{18}$ B. Yotsombat et al., J. Phys. D: Appl. Phys. 34(12), 1928 (2001).

${ }^{19}$ H. Rosenthal, I. Beilis, S. Goldsmith, and R. L. Boxman, J. Phys. D: Appl. Phys. 29(5), 1245 (1996).

${ }^{20}$ A. Kramida, Y. Ralchenko, and J. Reader, Spectral Line Database, http:// physics.nist.gov/asd

${ }^{21}$ R. L. Kurucz and B. Bell, Spectral line database, http://www.cfa.harvard. edu/amp/ampdata/kurucz23/sekur.html

${ }^{22}$ E. Meinders, Physica B+C 84, 117 (1976).

${ }^{23}$ A. G. Shenstone, J. Res. Nat. Bur. Stand. 79A(3), 497 (1975).

${ }^{24}$ A. R. Striganov and N. S. Sventitskii, Tables of Spectral Lines of Neutral and Ionized Atoms (Plenum, New York, Washington, 1968).

${ }^{25}$ A. N. Zaidel, V. K. Prokof'ev, and S. M. Raiskii, Tables of Spectrum Lines (Plenum, New York, Washington, 1970).

${ }^{26}$ A. A. Logatchev et al., in Proceedings of the XXIVth International Symposium on Discharges and Electrical Insulation in Vacuum, ISDEIV (Braunschweig, Germany, 2010).

${ }^{27} \mathrm{~S}$. G. R. Methling et al., in Proceedings of the XXVIth International Symposium on Discharges and Electrical Insulation in Vacuum, ISDEIV (Mumbai, India, 2014).

${ }^{28}$ S. A. Popov et al., IEEE Trans. Plasma Sci. 37(8), 1419 (2009).

${ }^{29}$ S. Goldsmith, Y. Bresler, and R. L. Boxman, J. Appl. Phys. 54(10), 5691 (1983).

${ }^{30}$ A. V. Batrakov et al., IEEE Trans. Plasma Sci. 39(6), 1296 (2011).

${ }^{31}$ A. Schneider et al., IEEE Trans. Plasma Sci. 39(11), 2844 (2011).

${ }^{32}$ W. D. Davis, J. Appl. Phys. 40(5), 2212 (1969).

${ }^{33}$ A. Anders, Phys. Rev. E 55, 969 (1997).

${ }^{34}$ A. Anders and G. Y. Yushkov, J. Appl. Phys. 91(8), 4824 (2002).

${ }^{35}$ R. Webster, J. Sherman, J. Jenkins, and D. Holmes, Univ. of Liverpool Arc Discharge Project Rep. ULAP-T43, 1976.

${ }^{36}$ R. B. S. Shalev and S. Goldsmith, in Proceedings of the XIth International Symposium on Discharges and Electrical Insulation in Vacuum, ISDEIV (Berlin, German Democratic Republic, 1984). 